\title{
Editorial
}

\section{Mathematical Modeling for Resources and Environmental Systems}

\author{
Y. P. Li, ${ }^{1}$ G. H. Huang, ${ }^{2}$ S. L. Nie, ${ }^{3}$ B. Chen, ${ }^{4}$ and X. S. Qin ${ }^{5}$ \\ ${ }^{1}$ MOE Key Laboratory of Regional Energy Systems Optimization, S-C Resources and Environmental Research Academy, \\ North China Electric Power University, Beijing 102206, China \\ ${ }^{2}$ Environmental Systems Engineering Program, Faculty of Engineering and Applied Science, University of Regina, \\ Regina, SK, Canada S4S 0A2 \\ ${ }^{3}$ College of Mechanical Engineering and Applied Electronics Technology, Beijing University of Technology, Beijing 100124, China \\ ${ }^{4}$ Faculty of Engineering \& Applied Science, Memorial University, St. John's, NL, Canada A1B 3X5 \\ ${ }^{5}$ School of Civil \& Environment al Engineering, Nanyang Technological University, Blk N1-01c-82, 50 Nanyang Avnue, \\ Singapore 639798
}

Correspondence should be addressed to Y. P. Li; yongping.li@iseis.org

Received 28 October 2013; Accepted 28 October 2013

Copyright (C) 2013 Y. P. Li et al. This is an open access article distributed under the Creative Commons Attribution License, which permits unrestricted use, distribution, and reproduction in any medium, provided the original work is properly cited.

Effective management and planning of resources and environmental systems have been of concerns in the past decades since contamination and resource-scarcity problems have led to a variety of impacts and liabilities. However, achieving a reasonable and efficient management strategy is difficult since many conflicting factors have to be balanced due to complexities of the real-world problems. In resources and environmental systems, there are a number of factors that need to be considered by planners and decision-makers, such as social, economic, technical, legislational, institutional, and political issues, as well as environmental protection and resources conservation. Moreover, a variety of processes and activities are interrelated to each other, resulting in complicated systems with interactive, dynamic, nonlinear, multiobjective, multistage, multilayer, and uncertain features. These complexities may be further amplified due to their association with economic consequences if the promises of expected targets are violated. Mathematical models are recognized as effective tools that could help examine economic, environmental, and ecological impacts of alternative pollution-control and resources-conservation actions, and thus aid planners or decision-makers in formulating costeffective management policies.

Therefore, to facilitate more robust management and planning of resources and environmental systems, innovative mathematical tools that are able to reflect various combinations of these complexities are desired. This special issue is devoted to provide a forum for facilitating discussions of emerging technologies for supporting decisions of resources and environmental management. It focuses on exposition of innovative methodologies for tackling challenges in modeling a variety of resources and environmental problems, as well as successful case studies. A number of state-of-the-art mathematical modeling studies related to resources and environmental systems are presented, which can help analyze the relevant information, simulate the related processes, implement pollutant mitigations, evaluate the resulting impacts, and generate sound decision alternatives.

The paper "Experimental study and modeling of fouling in immersed membrane bioreactor operating in constant pressure filtration" by M. Hosseinzadeh et al. proposed a new mathematical model to predict fouling in an airlift immersed membrane bioreactors. With experimental verification, the results showed that the ultimate filtration resistance and the initial rate of cake formation are more sensitive to the aeration rate at lower superficial velocities. It was also found that the ultimate filtration resistance has a linear relation with mixed liquor suspended solid (MLSS) concentration. 
The paper "Hydrological design of nonstationary flood extremes and durations in Wujiang River, south china: changing properties, causes, and impacts" by X. $\mathrm{H}$. Chen et al. analyzed the flood duration frequency (QDF) using annual maximum streamflow series of 1-10-day durations observed at Pingshi and Lishi stations in Wujiang River, southern China. The trends and change point of annual maximum flood flow and flood duration were also investigated by statistical tests. The results indicated that the annual maximum flood flow has a marginally increase trend, whereas the flood duration exhibits a significant decrease trend at the 0.10 significant level. A nonstationary frequency analysis taking account of change point in the data series is highly recommended for future studies.

The paper "Application of MM5-CAMx-PSAT modeling approach for investigating emission source contribution to atmospheric $\mathrm{SO}_{2}$ pollution in Tangshan, northern China" by L. Li et al. introduced an integrated MM5-CMAx-PSAT modeling approach to identify the variation of emission contribution from each modeling grid to regional and urban air quality per unit emission rate change. The method was applied to a case study in Tangshan, a typical industrial region in northern China. Principal component analysis, canonical correlation analysis, and Pearson correlation analysis indicated that there was an obvious negative correlation between the grid-based variation of emission contribution to regional air quality and planetary boundary layer height as well as wind speed, while terrain data presented insignificant impacts on emission contribution variation.

The paper "Numerical solution of advection-diffusion equation using a sixth-order compact finite difference method" by G. Gurarslan et al. conducted a study to produce numerical solutions of one-dimensional advection-diffusion equation using a sixth-order compact difference scheme in space and a fourth-order Runge-Kutta scheme in time. The suggested scheme was a very accurate and relatively flexible solution approach in solving the contaminant transport equation for $\mathrm{Pe} \leq 5$. The accuracy and validity of the numerical model were verified through the presented results and the literature. The computed results showed that the use of the current method in the simulation is very applicable for the solution of the advection-diffusion equation.

The paper "Numerical study on initial field of pollution in the Bohai Sea with an adjoint method" by C. H. Wang et al. introduced a marine ecosystem dynamical model to simulate the dispersion process of the pollution in the Bohai Sea, where routine monitoring data were assimilated to study the initial field of pollution by using the adjoint method. The model could help reduce the misfit between inversion results and observations significantly and make the simulated results closer to reality. The results illustrated that the adjoint method can make use of the existing observations to the maximum extent, where not only the values and locations of the observations but also the sampling time can be taken into consideration.

The paper "Simulation of multiphase flow of the oilwater separation in a rotating packed bed for oil purification" by X. J. Zhang et al. investigated the optimal operating conditions and configurations on the hydraulic performance of rotating packed bed (RPB). The operating conditions and configuration on the hydraulic performance of the RPB are investigated. The results indicated that the separation efficiency of high gravity rotary device rotating oil purifier is affected by the configuration, especially the layout of liquid inlet, oil outlet, and water outlet in the RPB. The mathematical models used in the study could contribute to a better understanding of the hydraulic characteristics of the RPB with the fast development of the computer technology.

The paper "Modified palmer drought severity index based on distributed hydrological simulation" by D. $\mathrm{H}$. Yan et al. modified the Palmer drought severity index (PDSI) based on a distributed hydrological model for Luanhe River basin, North China. The proposed method could not only reflect the spatial heterogeneity of regional drought and improve the physical mechanism of PDSI but also provide technical support for comprehensive understanding of drought and effective preventing and relieving of drought disasters.

The paper "Effect of land use and climate change on runoff in the Dongjiang basin of south china" by $\mathrm{Y}$. $\mathrm{H}$. He et al. identified the quantitative impacts of land use and climate change on the runoff through soil conservation service (SCS) monthly model. With the real case study for four subbasins in the Dongjiang basin of south China, their results disclosed that approximately $20 \sim 30 \%$ runoff change was contributed by land-use modification. Besides, the effect of climate change on runoff variation had a different inner-annual distribution even under same annual precipitation.

The paper "Guidance of autonomous amphibious vehicles for flood rescue support" by S. Ragi et al. advanced a pathplanning algorithm to guide autonomous amphibious vehicles (AAVs) for flood rescue support missions. An approximation method called nominal belief-state optimization (NBO) was proposed for solving the partially observable Markov decision process (POMDP) problems. Results showed that the NBO technique outperforms the greedy approach significantly.

The paper "Nonsmooth dynamic behaviors inherited from an ecohydrological model: mutation, bifurcation, and chaos" by M. Lin et al. advanced an ecohydrological model with the pulsed atmospheric forcing to analyze the nontrivial dynamic behaviors in a coupled ecohydrological system qualitatively and numerically. The developed mathematical model could help identify existence of multiple stationary states, study parameter bifurcation, and obtain the chaotic characteristic of the system state under some specific parameters.

The paper "Analysis of variation trends in precipitation in an upstream catchment of Huai River" by P. Shi et al. analyzed the variation trends in precipitation based on the linear regression method and Mann-Kendall test. With real application in Shaying River catchment, the upstream of the Huai River, China, the results suggested that the changing trends in precipitation for different time series in the whole catchment were relatively complex and not obvious. The annual precipitation series showed positive trends in the northern region and negative trends in the southern region.

The paper "Detecting abrupt change of streamflow at Lintong station of Wei River" by J. J. Fan et al. introduced two methods of moving approximate entropy and moving 
permutation entropy to analyze the abrupt year of the daily river runoff at Lintong station of Wei River in Loess Plateau, China. With the analysis of hydrological characters of different stages, the result showed that the proposed methods are useful for analyzing runoff series and identifying points of abrupt change. The results also disclosed that the attribution of abrupt change at the Lintong runoff series was primarily due to a number of factors such as reduced precipitation, increased water-conservancy project, boosted water consumption, decreased groundwater table, and raised evaporation.

The paper "A sustainability-oriented multiobjective optimization model for siting and sizing distributed generation plants in distribution systems" by G. Chen et al. proposed a multiobjective optimization model for siting and sizing distributed generation plants in distribution systems. With numerical study based on the modified IEEE 33-node system, the results indicated that benefits for environmental sustainability of using DG plants can be effectively reflected by the proposed model, which helps the planner to make rational decision towards sustainable development of the distribution system.

The paper "A model for urban environment and resource planning based on green GDP accounting system" by L. Y. $\mathrm{Xu}$ et al. proposed a model to identify the key factors in urban environment and resources regulation based on a green GDP accounting system. With real application in the City of Wuyishan, China, the results showed that energy use was a key factor that influenced the urban environment and resources development. It was also found that biodiversity and air quality were the most sensitive factors that influenced the value of green GDP in the study city. The proposed model is helpful for decision-makers in improving the urban environment and resources planning and promoting sustainable development.

Although many simulation and optimization models are developed for management and planning of resources and environmental systems, it is still difficult for decisionmakers to gain an in-depth insight into the tradeoffs when uncertainties exist in many system components and their interrelationships. There are many sources of uncertainty in modelling resources and environmental systems due to complexities in parameter estimation, data availability, and model structure. In detail, uncertainties may be derived from random feature of many related natural processes as well as errors in estimated modeling parameters; uncertainties can also arise due to human-induced imprecision or fuzziness, such as lack of available data and biased judgment (or preferences) in assigning priority factors (weighting levels) to multiple management objectives. Moreover, uncertainties may exist in multiple levels: vagueness and/or impreciseness in the outcomes of a random sample and randomness and/or fuzziness in the lower and upper bounds of an interval. The various uncertainties that exist in real-world resources and environmental systems have essentially placed them beyond the conventional mathematical methods. Thus, simulation and optimization methodologies that can reflect uncertainties are desired for enhancing applicability of the modeling efforts.
The paper "A hybrid stochastic-interval analytic hierarchy process approach for prioritizing the strategies of reusing treated wastewater" by L. Jing et al. proposed a hybrid stochasticinterval analytic hierarchy process (SIAHP) approach for prioritizing the strategies of reusing treated wastewater. SIAHP can address uncertainty in group decision making by integrating interval judgment, probabilistic distribution, lexicographic goal programming, and Monte Carlo simulation. A case study related to wastewater treatment plant effluent reuse was conducted to demonstrate that the SIAHP approach can aid group decision making by accommodating linguistic information and dealing with insufficient information or biased opinions.

The paper "Modelling snowmelt runoff under climate change scenarios in an ungauged mountainous watershed, northwest China" by Y. G. Ma et al. introduced an integrated modeling system for analyzing the impact of climate change on snowmelt runoff in Kaidu Watershed, Northwest China. The system coupled Hadley Centre Coupled Model version 3 (HadCM3) outputs with snowmelt runoff model (SRM), where statistical downscaling model (SDSM) was used to downscale coarse outputs of HadCM3. Different scenarios were considered for analyzing the climate change impact on snowmelt flow in the Kaidu Watershed under uncertainty. The results indicated that watershed hydrology would alter under different climate change scenarios, which could provide useful decision support for water resources management in the arid region.

The paper "Generalized likelihood uncertainty estimation method in uncertainty analysis of numerical eutrophication models: take BLOOM as an example" by Z. J. Li et al. introduced an eutrophication and algal bloom model BLOOM for investigating the applicability of the GLUE method to quantify the uncertainty in numerical eutrophication models. The results demonstrated that GLUE is effective for uncertainty analysis of complex dynamic ecosystem models. The method was found to be able to provide a solid foundation for the use of the model predictions in decision making.

The paper "An inventory-theory-based inexact multistage stochastic programming model for water resources management" by M. Q. Suo et al. introduced one integrated optimization method to address multiple forms of uncertainties incorporating inventory theory within an inexact multistage stochastic programming framework. The developed method can not only handle uncertainties represented as probability density functions and discrete intervals but also reflect dynamic features of system conditions under different water flow levels within a multistage context. The model is helpful for decision-makers in seeking optimal water transfer schemes when they are facing tradeoff problems with high complexities.

The paper "RSW-MCFP: a resource-oriented solid waste management system for a mixed rural-urban area through Monte Carlo simulation-based fuzzy programming" by $\mathrm{P}$. Li et al. introduced a resource-oriented solid waste management system for a mixed rural-urban area through Monte Carlo simulation-based fuzzy programming. The developed system was tested by a real-world case with consideration of various resource-oriented treatment technologies and the associated 
uncertainties. The results indicated that the communitybased biocoal and household-based $\mathrm{CH}_{4}$ facilities are necessary and become predominant in the waste management system.

The paper "Suitable environmental flow release criteria for both human and riverine ecosystems: accounting for the uncertainty of flows" by J. Tang et al. proposed a new method to determine the optimal environmental flow (eflow) release criteria under flow uncertainty accounting for both the human and riverine ecosystem needs. Scenariotree analysis method was utilized to generate the possible scenarios of flows and the range of variability approach (RVA) was refined by incorporating the uncertain flows. With the real application to Tang River of northern China and comparison of the previous method, the results indicated that the advanced can get more suitable criteria that are suitable for both human and riverine ecosystems.

The paper "Uncertainty assessment of hydrological frequency analysis using bootstrap method" by Y.-M. Hu et al. put forward a bootstrap-based method to analyze the impact of sampling uncertainty on hydrological design value. Using bootstrap resampling technique, a large number of bootstrap samples were constructed from the original flood extreme observations; the corresponding design value or quantile was estimated for each bootstrap sample, such that the sampling distribution of design value was constructed; based on the sampling distribution, the uncertainty of quantile estimation could be quantified. Compared with the conventional deterministic approach, the proposed method improved the estimation and evaluated quantitatively the uncertainty of estimation for the design of hydraulic engineering works.

The paper "Applying an extended fuzzy parametric approach to the problem of water allocations" by T. Y. Xu and X. S. Qin advanced an extended fuzzy parametric programming (EFPP) model for planning water resources allocation problems under uncertainty. A numerical example and a water resources management case were used to demonstrate the applicability of the proposed method. The obtained results could assist decision-makers in understanding the balance between the system benefit and system-failure risk. By comparing with fuzzy chance-constrained programming model, EFPP could better reflect uncertainties and generate more optimal solutions and had a potential to be applied to the other environmental problems.

The paper "Development of optimal water-resources management strategies for Kaidu-kongque watershed under multiple uncertainties" by Y. Zhou et al. advanced an intervalstochastic fractile optimization (ISFO) model for supporting water resources management under coupled forms of probability and possibility. With real application in Kaidu-kongque watershed, China, the model was found to be able to help decision-makers alleviate the water supply-demand conflict and achieve better socioeconomic and ecological sustainability. The results suggested that this hybrid technique is effective and can be extended to other environmental problems that involve policy analysis and uncertainty reflection.

The paper "Bilevel multiobjective programming applied to water resources allocation" by S. Q. Fang et al. proposed a bilevel mutiobjective linear programming (BLMOLP) model for water resource allocation. Fuzzy goal programming approach was employed to minimize the group regret of degree of satisfactions for all decision-makers. The proposed method was also applied to a case study for water resources allocation in Wuwei Basin, China. Based on analysis of multiple scenarios, the model was found to be able to balance the benefits among all regions and sections according to the priority of the upper level decision-makers.

The paper "An interval-parameter fuzzy linear programming with stochastic vertices model for water resources management under uncertainty" by Y. Han et al. introduced an integrated optimization method for handling uncertainties presented as interval parameter with stochastic vertices in both of the objective functions and constraints. A hybrid intelligent algorithm based on genetic algorithm and artificial neural network was proposed to solve the formulated model. The developed method was applied to water resources allocation in Beijing, China. The results indicated that reasonable solutions were obtained, which could help water resources managers to not only make decisions of water allocation but also gain insight into the tradeoffs between environmental and economic objectives.

In summary, the effective mathematical models for managing resources and environmental systems are becoming one of the most important goals pursued by governments, industries, communities, and researchers. These 25 papers formulated a variety of mathematical models for supporting natural resources allocation, flooding prevention and control, environmental pollution control, ecological protection, and sustainable development improving, where a number of innovative perspectives and findings are advanced. These mathematical models can (i) describe complex environmental processes and interactions, characterize the spatial and temporal variations, and predict the fate and transport of the contaminants; (ii) assess potential risks existing in various resources-related activists and the associated socioeconomic and environmental impacts under a variety of system conditions; (iii) generate sound decision alternatives for generating desired policies that target on more effective resources and environmental management. This special issue reports the latest research results in mathematical modeling for resources and environmental systems.

\section{Acknowledgment}

We wish to extend our thanks to those who contributed to this issue.

Y. P. $\mathrm{Li}$

G. H. Huang

S. L. Nie

B. Chen

X. S. Qin 


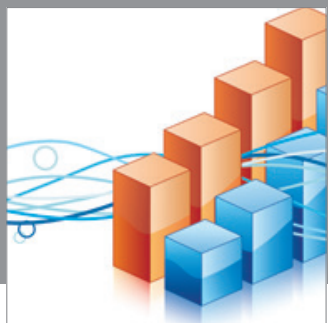

Advances in

Operations Research

mansans

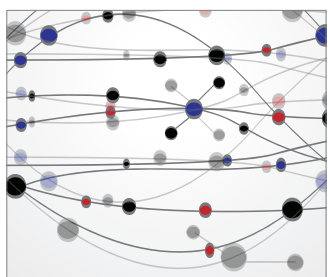

The Scientific World Journal
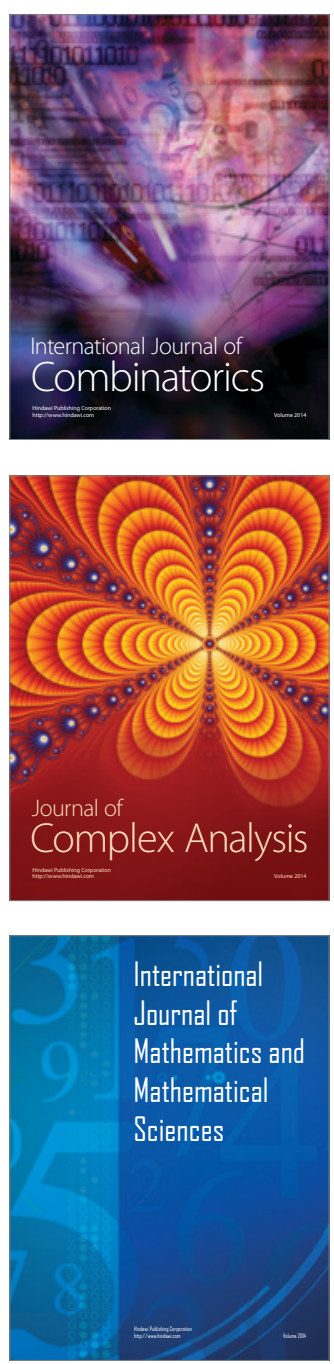
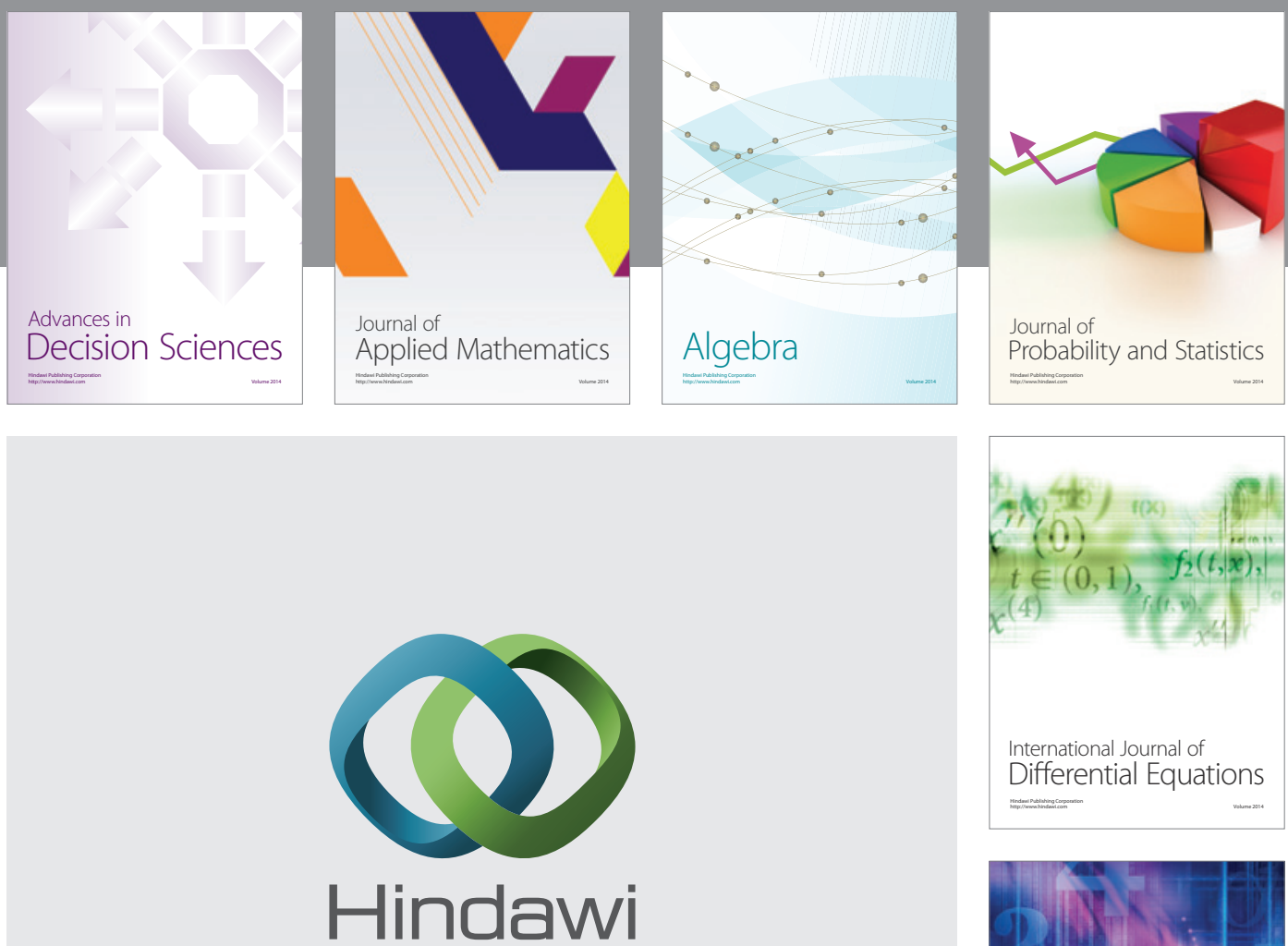

Submit your manuscripts at http://www.hindawi.com
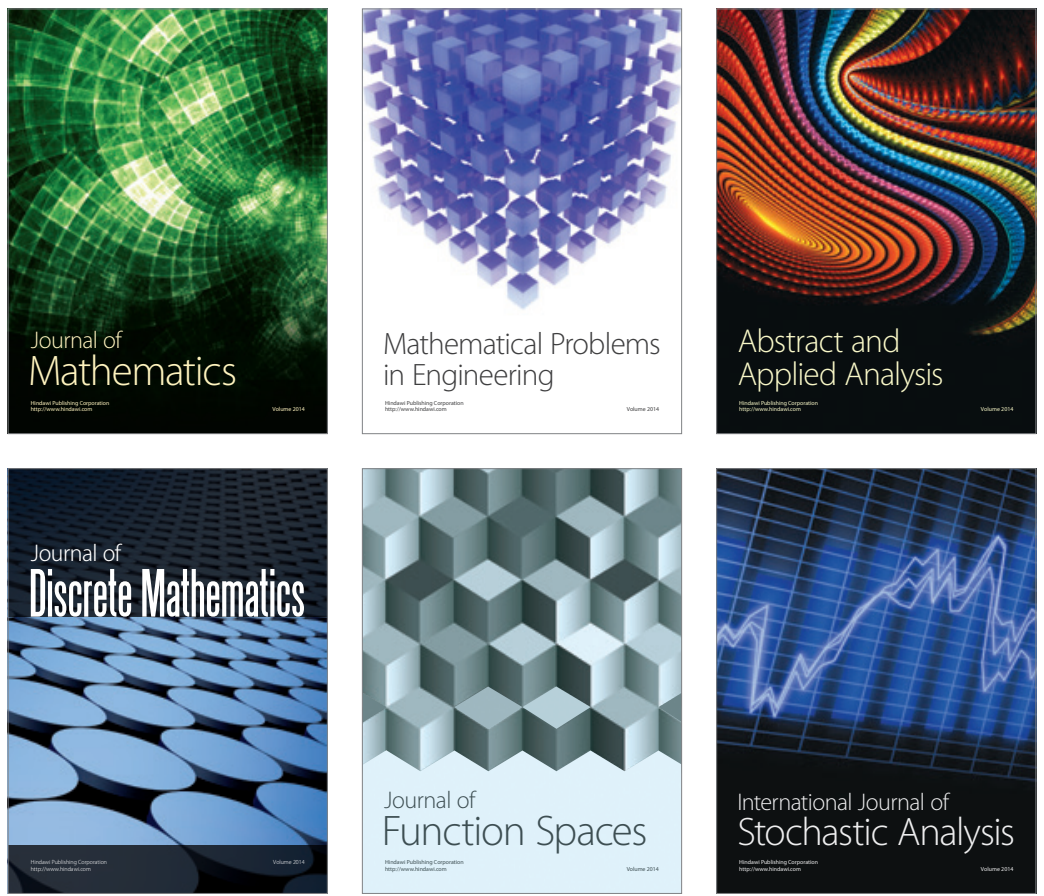

Journal of

Function Spaces

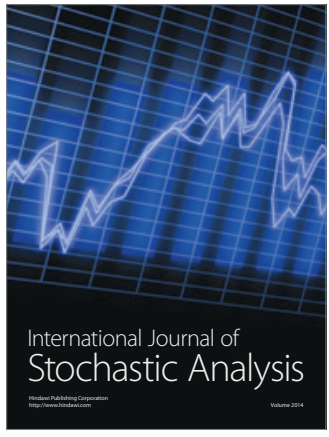

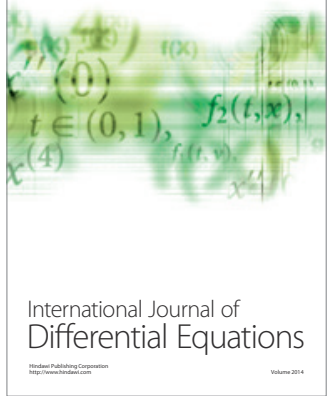
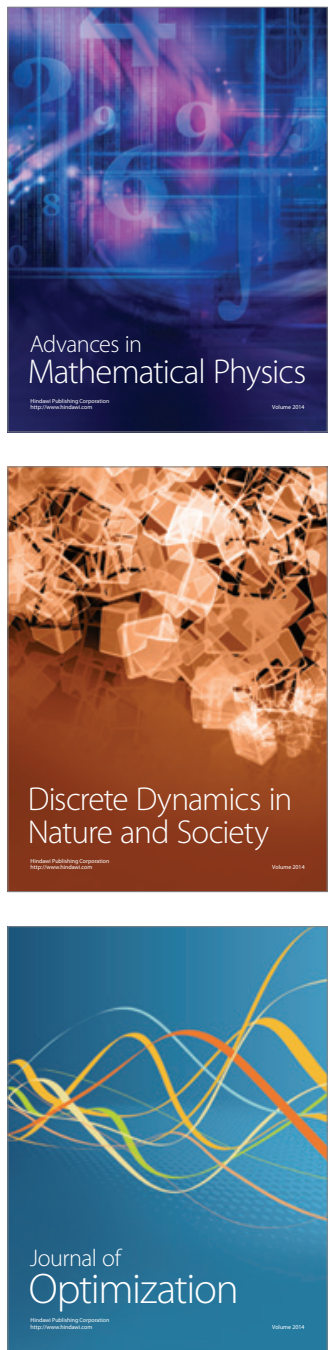